\title{
Uso del Modelo Autorregresivo de Duración Condicional para predecir la caída del dólar en el mercado cambiario colombiano
}

\author{
Recibido: octubre 26, 2019 - Aprobado: febrero 12, 2020
}

Doi: https://doi.org/10.12804/revistas.urosario.edu.co/economia/a.8280

\author{
Héctor Fabio Gallego Escudero* \\ Omar Alexander Ríos Saavedra ${ }^{\dagger}$
}

\section{Resumen}

El objetivo fundamental del modelo autorregresivo de duración condicional (ADC) es el modelamiento de series de tiempo con periodos no equidistantes. Dada la naturaleza leptocúrtica de los retornos de la tasa representativa del mercado (TRM) y el comportamiento de las duraciones asociado a estos, se usó una distribución de Rayleigh con transmutación, que permite aproximar de forma ajustada a una distribución de colas pesadas, coherente con este hecho estilizado en los retornos. Se concluye que el tiempo transcurrido entre caídas del dólar, en promedio, se encuentra entre 3 y 6 días.

Palabras clave: autorregresivo de duración condicional; periodos no equidistantes; Rayleigh; Transmutación; TRM.

JEL: Business Fluctuations; Cycles E32.

* Universidad del Valle. Correo electrónico: hector.gallego@correounivalle.edu.co

† Universidad del Valle. Correo electrónico: omar.rios@correounivalle.edu.co

Para citar este artículo: Gallego Escudero, H. F., \& Ríos Saavedra. (2020). Uso del Modelo Autorregresivo de Duración Condicional para predecir la caída del dólar en el mercado cambiario colombiano. Revista de Economía del Rosario, 23(2), 1-21. https://doi.org/10.12804/ revistas.urosario.edu.co/economia/a. 8280 


\title{
Use of the Autoregressive Conditional Duration Model to predict the dollar fall in the colombian Exchange Market
}

\begin{abstract}
The fundamental objective of the Autoregressive Conditional Duration (acd) model is the modeling of time series with non-equidistant periods. Given the leptokurtic nature of trm returns and the durations behavior associated with them, a Rayleigh Distribution with transmutation is used, which allows approaching in an adjusted form to a heavy tail distribution, coherent with this stylized fact in returns. It is concluded that the time elapsed between dollar falls, on average, is between 3 and 6 days.
\end{abstract}

Keywords: Autoregressive Conditional Duration; non-equidistant periods; Rayleigh; Transmutation; TRM.

JEL: Business Fluctuations; Cycles E32.

\section{Uso do Modelo Auto-regressivo de Duração Condicional para predizer a queda do dólar no mercado cambiário colombiano}

\section{Resumo}

O objetivo fundamental do modelo Auto-regressivo de Duração Condicional (ADC) é o modelamento de séries de tempo com períodos não equidistantes. Dada a natureza leptocúrtica dos retornos da TRM e o comportamento das durações associados a estes, se usou uma Distribuição de Rayleigh com transmutação, que permite se aproximar de forma ajustada, a uma distribuição de colas pesadas, coerente com este facto estilizado nos retornos. Se conclui que, o tempo transcorrido entre quedas do dólar, em média, encontra-se entre 3 e 6 dias.

Palavras-chave: Auto-regressivo de Duração Condicional; períodos não equidistantes; Rayleigh; Transmutação; TRM.

JEL: Business Fluctuations; Cycles E32. 


\section{Introducción}

La tasa de cambio representativa del mercado (TRM) colombiano es la cantidad en pesos por dólar estadounidense, que se calcula con base en las operaciones de compra y venta de divisas entre intermediarios financieros que realizan transacciones en el mercado cambiario colombiano (Banco de la República, 1991-2020). La variación del peso respecto al dólar es importante debido a que este es un factor determinante en la valorización o desvalorización del peso colombiano. Además, tiene implicaciones en la economía interna y externa como el impacto en los precios de productos importados, que a su vez tienen efecto sobre la inflación, las negociaciones con divisas, y el impacto en la competitividad de empresas nacionales respecto a competidores internacionales.

Se han realizado distintos trabajos sobre el modelado y pronóstico de la TRM, evaluando diversos factores influyentes en el precio del dólar. Se destacan, entre otros trabajos, el de Alonso y Patiño (2005), quienes estudiaron los diferenciales en la productividad sobre la tasa de cambio nominal, utilizando modelos econométricos de precio fijo y el enfoque de Balassa-Samuelson. Estos modelos indican que un incremento en la oferta monetaria genera una salida de capital y, en consecuencia, una depreciación de la tasa de cambio. Santana (2006) utilizó un modelo de series temporales con redes neuronales aplicado a la variación de la inflación basada en el Índice de Precios al Consumidor. Henao y Rivera (2006) y Jalil y Misas (2007) utilizaron modelos de series temporales con redes neuronales para pronosticar el precio del dólar.

Cuando el dólar sube, el precio de los productos importados aumenta y, en consecuencia, aumenta la inflación; mientras que cuando el dólar baja, al reducir la competitividad de las empresas nacionales, estas se ven obligadas a reducir sus costos de producción, lo que termina causando despidos y desaceleración económica (Bonifaz, 2004).

Dadas estas implicaciones, es importante modelar el tiempo en que ocurre la variación del dólar. En este documento, se propone un modelo que permite describir un suceso como la baja abrupta del precio del dólar respecto al peso en el mercado cambiario colombiano, que constituye un comportamiento no regular en la periodicidad de la serie de tiempo.

Un suceso puede ser definido como una transacción, un cambio en el precio excediendo cierto monto o cualquier característica de interés a ser investigada (Cheung, 2014). En otras palabras, un suceso es un acontecimiento observable que tiene impacto sobre una economía. Engle y Russell (1997) propusieron un modelo que describe el tiempo transcurrido entre sucesos económicos que tienen una estructura de dependencia. El modelo 
Autorregresivo de Duración Condicional (ACD por sus siglas en inglés) tiene como objetivo fundamental describir el comportamiento de series de tiempo cuyos periodos no son equidistantes, ya que las series de tiempo a modelar están condicionadas por el suceso observado.

Distintas aplicaciones en el modelado de datos financieros mediante el modelo ACD, han sido presentadas por autores como Tse y Yang (2012) para modelar la volatilidad intradía de acciones cotizadas en la bolsa de valores de Nueva York, específicamente, la volatilidad integrada condicionada a cierta información de la bolsa. Uno de los resultados más relevantes de la investigación de estos autores indica que las volatilidades anualizadas de las compañías General Electric y TJX presentaron grandes fluctuaciones intradía. Similar al trabajo de Tse y Yang (2012), Liu y Tse (2015) modelaron el valor en riesgo intradía, argumentando que debido a la regla de acceso al mercado, toda orden debe pasar por un control de riesgo antes de realizarse cualquier transacción. El modelo fue aplicado a existencias del S\&P 500, examinando el patrón del valor en riesgo que mostró que, para cierto momento del día, el valor de riesgo era menor (entre las 11:00 y 14:30).

Pyrlik (2013) modela el comportamiento de desplomes financieros del promedio industrial Dow Jones mediante el enfoque ACD. La obtención de las duraciones las obtuvo mediante un valor crítico en las rentabilidades diarias asociadas al Dow Jones, las cuales, según el autor, se fijaron en -3\%. En su estudio, el autor muestra la capacidad del modelo para predecir el tiempo transcurrido hasta observar un desplome.

Los desplomes financieros son un ejemplo de eventos cuya ocurrencia presenta una duración no periódica. Estos tienen una gran variedad de consecuencias económicas negativas que van desde pérdidas de ingreso persona, hasta bancarrotas masivas y recesiones (Farmer, 2012). Las duraciones no periódicas dan lugar a tiempos transcurridos de manera aleatoria por lo que para su estudio se utilizan metodologías estadísticas propias del análisis de tiempo de vida.

En este estudio, se tiene como suceso de interés la caída del dólar, modelando el tiempo de duración transcurrido entre dos momentos de caída, apoyado en la variación del precio del dólar en pesos colombianos mediante los log-retornos de dicho precio. La caída del dólar, de forma similar a un desplome financiero, da lugar a una duración no periódica de la serie de tiempo de la TRM. Dicha caída se presenta cuando la rentabilidad del precio del dólar presenta un valor negativo, que indica que el precio del dólar en un día cualquiera es significativamente menor respecto al precio del dólar el día anterior. Esta caída del precio del dólar, al presentar una duración no periódica, corresponderá a duraciones transcurridas de manera aleatoria. 
Dada la naturaleza aleatoria de las duraciones entre caídas del dólar, estas presentan un comportamiento de asimetría marcada y valores extremos, por ello, en este estudio se propone el uso de la distribución de Rayleigh transmutada. Esta distribución permite capturar la asimetría del tiempo transcurrido entre caídas del dólar y las duraciones extremas.

La estructura del documento es la siguiente: en la primera sección se describen el modelo, los datos y la estimación; en la segunda se muestran los resultados de la estimación del modelo y en la tercera se discuten las conclusiones.

\section{Metodología}

\subsection{El modelo Autorregresivo de Duración Condicional}

La duración entre eventos en agentes económicos ha sido ampliamente considerada en estudios teóricos y empíricos desde 1970 (Pyrlik, 2013). El análisis de supervivencia generalmente asume independencia en los eventos estudiados y, en consecuencia, también las duraciones entre eventos son independientes. Sin embargo, Engle y Russell (1997) introdujeron un modelo en el cual tanto los eventos estudiados, como la duración entre ellos son interdependientes temporalmente. El modelo, se define así:

Sea $x_{i}=t_{i}-t_{i-1}$, la duración entre dos eventos críticos en un tiempo $t_{i} \mathrm{y}$ $t_{i-1}$, respectivamente. Las duraciones $x_{i}$, se describen mediante un modelo multiplicativo, tal que:

$$
x_{i}=\phi_{i} \xi_{i}
$$

Donde $\phi_{i}$ es la $i$-ésima duración condicional del modelo, y $\xi_{i}$ es la $i$-ésima duración estocástica. Las duraciones estocásticas se asumen independientes e idénticamente distribuidas; además, $\xi_{i}>0$ con valor esperado $E\left[x_{i}\right]=\mu$.

De este modelo, es conveniente derivar el valor esperado de la duración $x_{i}$, condicionado a la información rezagada $z_{i}=\left\{x_{j}, \xi_{j} \phi_{i}\right\}_{j<i}$, así (Pyrlik 2013):

$$
E\left[x_{i} \mid z_{i}\right]=E\left[\phi_{i} \xi_{i} \mid z\right]=\phi_{i} E\left[\xi_{i}\right]=\phi_{i} \mu
$$

Dado el valor esperado condicional en (2), es posible escribir el modelo en (1), en términos de sus componentes estandarizados:

$$
x_{i}=\psi_{i} \in
$$


En (3), la duración condicional $\psi_{i}=\phi_{i} \mu$ y la duración estocástica $\epsilon_{i}=\xi_{i} / \mu$ tienen valor esperado $E\left[\in_{i}\right]=1 . \in_{i}$ es llamada duración estocástica estandarizada. El modelo presentado en (3) es definido como el modelo ACD de orden $(p, q)$, tal que:

$$
\begin{gathered}
x_{i}=\psi_{i} \in_{i} \\
\psi_{i}=\omega+\sum_{j=1}^{p} \alpha_{i} x_{i-j}+\sum_{j=1}^{q} \beta_{i} \psi_{i-j}
\end{gathered}
$$

En el modelo, $\omega$ es el intercepto, $\sum_{j=1}^{p} \alpha_{j} x_{i-j}$ es el componente autorregresivo y $\sum_{j=1}^{q} \beta_{j} \psi_{i-j}$ es el componente de duración condicional. En particular, el modelo ACD $(1,1)$, tiene la siguiente forma para $\psi_{i}$ :

$$
\psi_{i}=\omega+\alpha_{1} x_{i}+\beta_{1} \psi_{i-1}
$$

El modelo ACD $(1,1)$ corresponderá a un proceso débilmente estacionario cuando $\alpha_{1}+\beta_{1}<1$.

Como se puede observar, la estructura matemática del modelo ACD es similar a la estructura del modelo GARCH introducido por Bollerslev (1986); por consiguiente, conserva muchas de sus propiedades. Algunas de estas propiedades para el modelo ACD $(1,1)$ son

$$
\begin{gathered}
E\left[x_{i}\right]=E\left[\psi_{i}\right]=\omega /\left(1-\left(\alpha_{1}+\beta_{1}\right)\right) \\
\operatorname{Var}\left(x_{i}\right)=E\left[x_{i}\right]-\left(E\left[x_{i}\right]\right)^{2} \\
\rho k\left(\in_{i}\right)=0
\end{gathered}
$$

$E\left[x_{i}\right]$ y $\operatorname{Var}\left(x_{i}\right)$ corresponden al valor esperado y varianza incondicionales de la duración $x_{i}$, respectivamente. La varianza incondicional de la duración $x_{i}$ está definida por

$$
\operatorname{Var}\left(x_{i}\right)=E\left[x_{i}^{2}\right]-\left(E\left[x_{i}\right]\right)^{2}=E\left[x_{i}\right]^{2} \frac{1-\beta_{1}^{2}-2 \alpha_{1} \beta_{1}}{1-\beta_{1}^{2}-2 \alpha_{1} \beta_{1}-2 \alpha_{1}^{2}}
$$

Las duraciones están asociadas a distribuciones asimétricas, que modelan tiempos de vida. Diversos autores, como Engle y Russell (1998), Tsay (2005) 
y Cheung (2014), han considerado distribuciones que modelan tiempos de vida tales como la exponencial, la gamma y la Weibull para las duraciones estocásticas. Asumir distribuciones de probabilidad para las duraciones estocásticas es importante en el proceso de estimación del modelo ACD por máxima verosimilitud.

Similar al comportamiento natural de las rentabilidades de la TRM, que dan lugar a una distribución de colas pesadas, los periodos no equidistantes entre caídas del dólar pueden presentar valores extremos. Dada esta implicación, se considera en este trabajo un modelo basado en la distribución de Rayleigh transmutada, la cual considera también una asimetría marcada y de cola larga. Este modelo permite capturar periodos largos entre caídas del precio del dólar.

\subsection{La distribución de Rayleigh transmutada}

Esta distribución es una generalización de la distribución de Rayleigh utilizando el mapa de transmutación de rango cuadrático introducido por Shaw y Buckley (2009). El mapa de transmutación de rango cuadrático tiene la siguiente forma: dadas dos distribuciones con un espacio muestral común y con funciones de distribución acumuladas $F_{X}(\cdot)$ y $G_{X}(\cdot)$. El mapa de transmutación de rango cuadrático está dado por

$$
G_{X}(x)=(1+\lambda) F_{X}(x)-\lambda F_{X}^{2}(x),|\lambda| \leq 1
$$

Donde $\lambda$ es el parámetro de transmutación.

A partir de (4) y de acuerdo con Merovci (2013), se tiene la siguiente definición de la distribución de Rayleigh transmutada: sea $X$ una variable aleatoria continua con distribución de Rayleigh de parámetro $\delta$. X seguirá una distribución de Rayleigh transmutada (TRayleigh) de parámetros $\delta$ y $\lambda$ si tiene una función de distribución acumulada y una función de densidad de probabilidades $F X(\cdot)$ y $f X(\cdot)$, respectivamente, tal que:

$$
\begin{gathered}
F_{X}\left(x[\delta, \lambda)=\left(1-\exp \left[-\frac{x^{2}}{2 \delta^{2}}\right]\right)\left(1+\lambda \exp \left[-\frac{x^{2}}{2 \delta^{2}}\right]\right)\right. \\
f_{X}\left(x\lceil\delta, \lambda)=\frac{x}{\delta^{2}} \exp \left[-\left(\frac{x^{2}}{2 \delta^{2}}\right)\right]\left(1-\lambda+2 \lambda \exp \left[-\left(\frac{x^{2}}{2 \delta^{2}}\right)\right]\right), x>0, \delta>0,|\lambda| \leq 1\right.
\end{gathered}
$$


Asumir una distribución de probabilidad relacionada con el análisis de tiempos de vida es importante en el modelo ACD, ya que este supuesto distribucional permite obtener una forma de estimación del modelo. El tiempo transcurrido entre caídas del dólar supone un comportamiento de asimetría marcada y cola larga; es decir, duraciones extremas, por lo que se asume la distribución de TRayleigh para realizar el proceso de estimación y predicción.

\subsection{Estimación del modelo y pronóstico}

El espaciado irregular de una serie de tiempo determinada por un suceso observado en una economía se define como una duración aleatoria. Para estimar el modelo propuesto se asume una distribución relacionada con el análisis de tiempo de vida. Esta distribución permite obtener una forma funcional, la cual indexa la información contenida en la serie de tiempo de las duraciones, que a su vez es extraída de la serie irregular determinada por el suceso observado.

La forma funcional que indexa la información contenida en las duraciones es la función de verosimilitud, que se construye mediante la función de densidad asociada a la distribución de probabilidad asumida. De este modo, la estimación del modelo se realiza mediante el método de máxima verosimilitud, a partir de la distribución asumida para las duraciones estocásticas. Dado que la función de densidad de las duraciones estocásticas $E_{i}$, es la siguiente:

$$
f\left(\epsilon_{i}\right)=\frac{\epsilon_{i}}{\delta^{2}} \exp \left[-\left(\frac{\varepsilon_{i}^{2}}{2 \delta^{2}}\right)\right]\left(1-\lambda+2 \lambda \exp \left[-\left(\frac{\varepsilon_{i}^{2}}{2 \delta^{2}}\right)\right]\right), \epsilon_{i}>0, \delta>0,|\lambda| \leq 1
$$

De acuerdo con la definición descrita por Merovci (2013), sobre la distribución de TRayleigh, y la definición del valor esperado de las duraciones estocásticas descrita en la sección 1.1., se tiene que:

$$
E\left[\epsilon_{i}\right]=\frac{1}{2} \delta \sqrt{\pi}(\lambda+\sqrt{2}(1-\lambda))=1
$$

De la expresión anterior, se puede escribir el parámetro $\delta$ en términos de $\lambda$, de modo que:

$$
\delta=\frac{2}{\sqrt{\pi}(\lambda+\sqrt{2}(1-\lambda))}
$$


Por lo anterior, con base en el trabajo de Engle y Russell (1998), y escribiendo $\epsilon_{i}=x_{i} / \psi_{i}{ }^{\prime}$ la función de verosimilitud del modelo ACD $(1,1)$ queda expresada así:

$$
L\left(\alpha_{1}, \beta_{1}, \omega, \lambda \mid x_{N(t)}\right)=\prod_{i=1}^{n} \frac{\left(x_{i} / \psi_{i}^{2}\right)}{\delta^{2}} \exp \left[-\left(\frac{\left(x_{i} / \psi_{i}\right)^{2}}{\delta^{2}}\right)\right]\left(1-\lambda+2 \lambda \exp \left[-\left(\frac{\left(x_{i} / \psi_{i}\right)^{2}}{\delta^{2}}\right)\right]\right)
$$

De la función de verosimilitud se obtiene la función de log-verosimilitud, descrita de la siguiente forma:

$$
\begin{aligned}
& \ell\left(\alpha_{1}, \beta_{1}, \omega, \lambda \mid x_{N(t)}\right) \\
& =\sum_{i=1}^{n} \ln \left(\frac{x_{i}}{\psi_{i}^{2} \delta^{2}}\right)-\frac{x_{i}^{2}}{\psi_{i}^{2} \delta^{2}}+\ln \left(1-\lambda+2 \lambda \exp \left[-\left(\frac{\left(\sum_{i=1}^{n} x_{i} / \psi_{i}\right.}{2 \delta^{2}}\right)\right]\right)
\end{aligned}
$$

En las funciones de verosimilitud y log-verosimilitud, $\psi_{i}$ contiene los parámetros $\omega, \alpha_{1}, \beta_{1}$. La función de log-verosimilitud y el estimador del modelo se construyeron mediante el software estadístico $\mathrm{R}$, con base en las funciones construidas por Tsay (2011). El objetivo de un modelo de series temporales es hacer pronósticos de observaciones futuras. Para el caso del modelo ACD, se realizará un pronóstico del tiempo que transcurre hasta un próximo evento crítico.

Para medir la precisión del pronóstico se cuenta con diferentes medidas como la raíz del error cuadrático medio (RMSE) o el error porcentual medio absoluto (MAPE). Si se tiene $l=1,2, \ldots, L$ pronósticos de futuras duraciones respecto a un origen $h$, definidas como $\left\{x_{h}(1), x_{h}(2), \ldots, x_{h}(L)\right\}_{\text {, , entonces la RMSE }}$ y el MAPE tienen la siguiente forma (Cheung 2014):

$$
\begin{gathered}
\text { RMSE }=\sqrt{\frac{1}{L} \sum_{l=1}^{L}\left(x_{h+1}-x_{h}(l)\right)^{2}} \\
M A P E=100 \frac{1}{L} \sum_{l=1}^{L}\left|\frac{x_{h+l}-x_{h}(l)}{x_{h+l}}\right|
\end{gathered}
$$




\section{Resultados}

De acuerdo con la metodología propuesta se muestran los resultados de la estimación y predicción del modelo ACD para la serie de días transcurridos entre bajas del dólar, así como los resultados del análisis de los residuales del modelo. La obtención del tiempo transcurrido entre bajas del dólar se realizó a partir de la serie de tiempo diaria de la TRM desde el 27 de noviembre de 1991 hasta el 22 de julio de 2019.

\subsection{Extracción de las duraciones}

Similar al trabajo de Pyrlik (2013), la extracción de las duraciones se realizó teniendo en cuenta los log-retornos del precio del dólar. En la figura 1, los log-retornos negativos indican una baja en el dólar; con base en estos, se establece el umbral: suceso crítico.
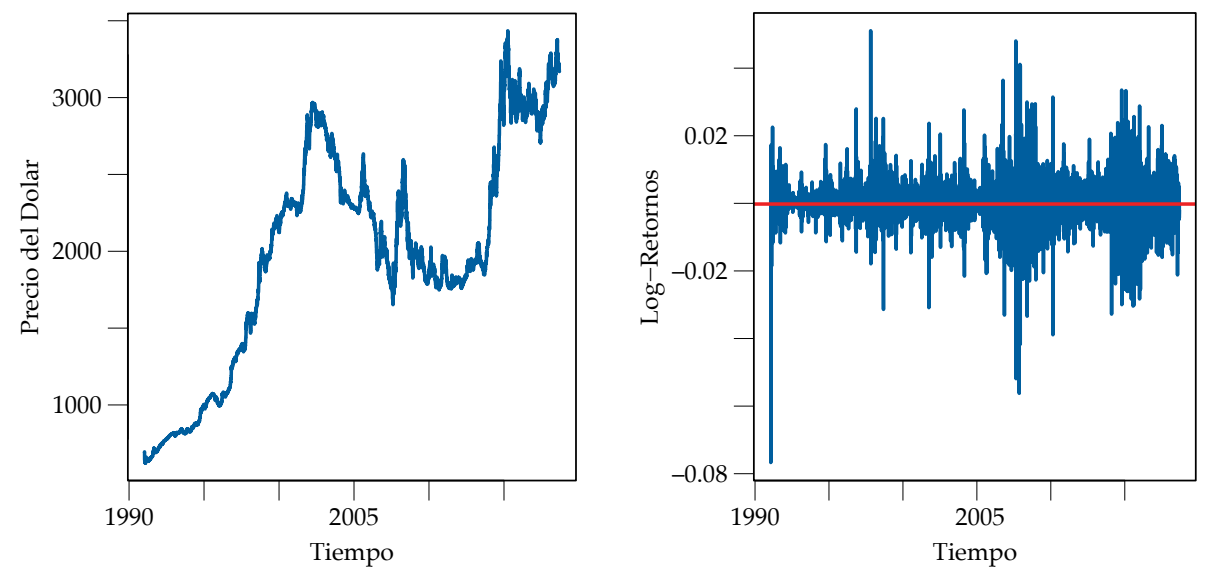

Figura 1. TRM y log-retornos

Con base en el umbral, se obtiene un subconjunto de la serie de tiempo de los log-retornos y las diferencias entre las fechas en las que suceden las caídas del dólar; estas diferencias son de la forma $x_{i}=t_{i} t_{i-1}$. Las diferencias entre las fechas corresponden al tiempo de duración entre caídas con las cuales se conforma una nueva serie de tiempo (figura 2).

Se realizaron pruebas de asimetría y curtosis para la serie de días transcurridos entre las caídas del dólar (Shapiro, Wilk \& Chen, 1968), las cuales 
mostraron que las duraciones presentan marcada asimetría y exceso de curtosis (tabla 1). De acuerdo con este resultado, la distribución TRayleigh es una alternativa para la estimación y pronóstico del modelo de duración.

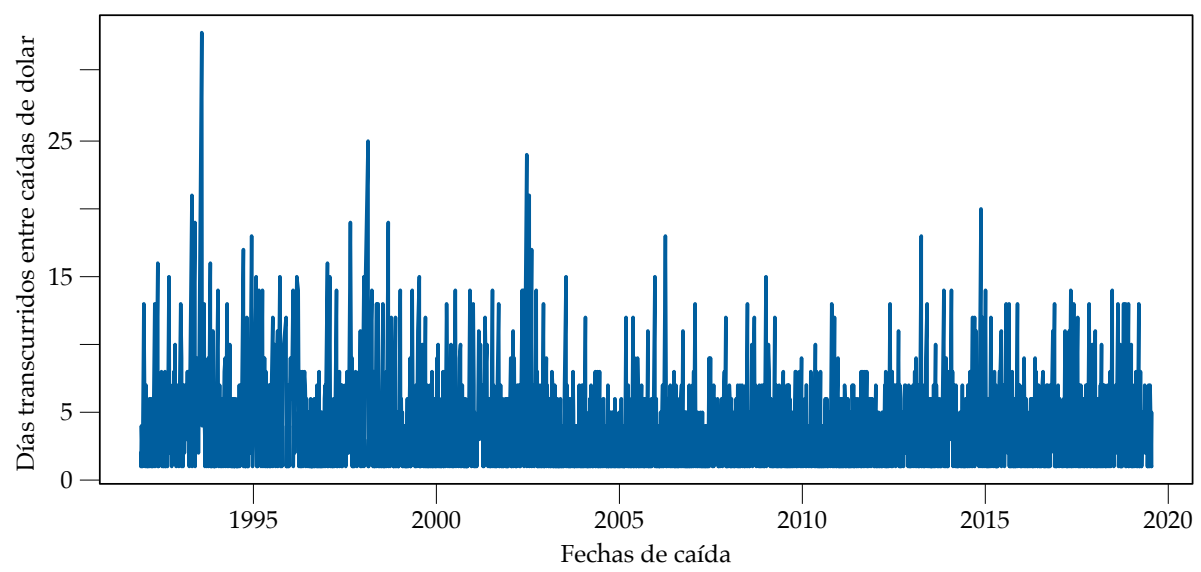

Figura 2. Serie de días transcurridos entre caídas del dólar

Tabla 1. Pruebas de asimetría y curtosis

\begin{tabular}{ccc}
\hline & Estadístico & Valor-p \\
\hline Asimetría & 2.2416 & $2.2 \mathrm{e}-16$ \\
Curtosis & 11.22087 & $2.2 \mathrm{e}-16$ \\
\hline
\end{tabular}

Para establecer el orden del modelo, es decir, cuántos rezagos se incluyen, se muestra la función de autocorrelación (figura 3), además del comportamiento del tiempo transcurrido entre caídas del precio del dólar (figura 4).

\subsection{Resultados de la estimación}

A continuación, se muestra la estimación por máxima verosimilitud del modelo ACD $(1,1)$ para la serie de días transcurridos entre bajas del dólar, mostrando la significancia individual de los parámetros estimados.

Las estimaciones de $\alpha_{1}$ y $\beta_{1}$ en la tabla 2 muestran que la suma de ellas es menor que 1 , indicando estacionariedad. La estimación de $\lambda$ corresponde a la estimación del parámetro de transmutación de la distribución de TRayleigh. Las estimaciones son significativas a un nivel de $\alpha=0.05$. 


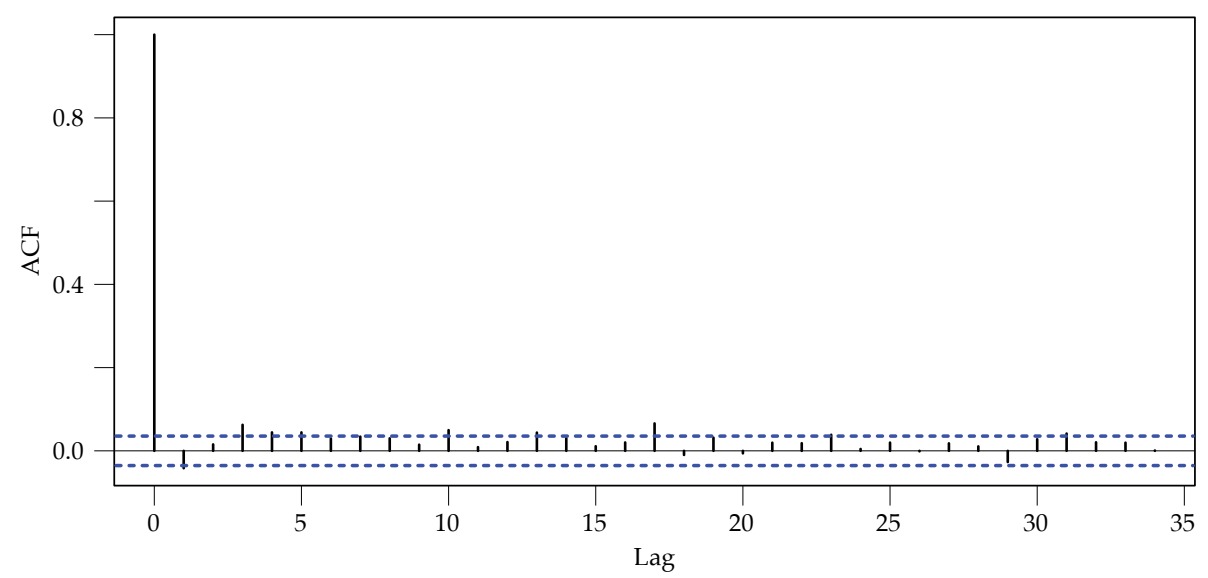

Figura 3. Función de Autocorrelación del número de días transcurridos entre bajas del dólar

Tabla 2. Estimación del modelo ACD $(1,1)$, con distribución de TRayleigh

\begin{tabular}{ccccc}
\hline & Estimate & Std. Error & $\mathrm{t}$-value & $\operatorname{Pr}>\mathrm{t}$ \\
\hline$\omega$ & 0.13 & 0.0345 & 3.7657 & 0.0002 \\
$\alpha$ & 0.04 & 0.0063 & 6.6986 & 0 \\
$\beta$ & 0.95 & 0.00915 & 103.6138 & 0 \\
$\lambda$ & 0.702 & 0.0239 & 29.3755 & 0 \\
\hline
\end{tabular}

\subsection{Residuales del modelo}

Se realizó el análisis de los residuales del modelo estimado en el que se estudió la independencia mediante la prueba de Ljung-Box, la cual mostró que con un estadístico de prueba $\chi^{2}=729.93$ con 766 grados de libertad y con un valor- $p$ de 0.821 , a un nivel de significancia de $\alpha=0.05$, no hay evidencia para rechazar independencia en los residuales del modelo estimado.

La definición del modelo ACD (p, q) asume que las duraciones estocásticas deben ser independientes e idénticamente distribuidas. Para mostrar la independencia de dichas duraciones estocásticas también se realizó un correlograma (figura 5).

\subsection{Resultados del pronóstico}

En esta sección, se muestran algunos resultados de pronósticos realizados con el modelo ACD $(1,1)$. 


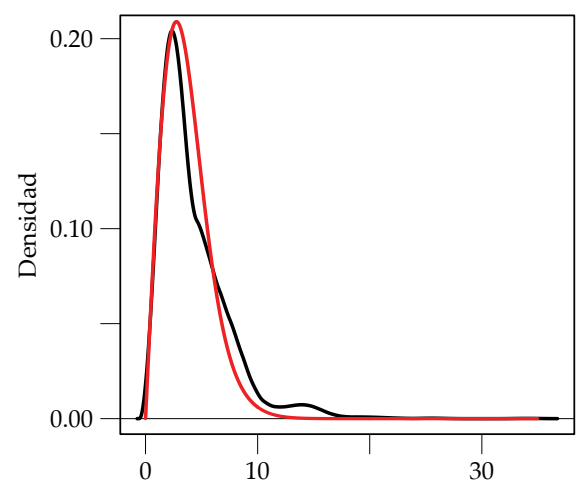

Días transcurridos entre caídas de dolar

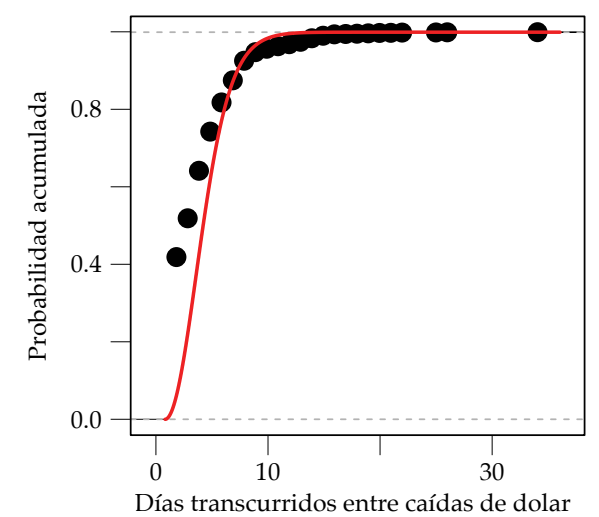

Duraciones

Figura 4. Comportamiento del número de días transcurridos entre bajas del dólar

$$
\begin{gathered}
\hat{x}_{i}=\widehat{\psi}_{i} \\
\widehat{\psi}_{i}=0.13+0.04 x_{i-1}+0.95 \widehat{\psi}_{i+1}
\end{gathered}
$$

Con dicha expresión, se obtienen la RMSE y el MAPE para cierto número de pronósticos. La función de pronóstico se construyó con base en la función de pronóstico presentada por Cheung (2014).

Tabla 3. RMSE y MAPE para distintos números de pronósticos

\begin{tabular}{ccc}
\hline Núm. Pronósticos (L) & RMSE & MAPE \\
\hline 1 & 3.76 & 375.61 \\
2 & 3.84 & 383.85 \\
3 & 3.92 & 391.99 \\
4 & 4 & 400.06 \\
\hline
\end{tabular}

La tabla 3 muestra que la RMSE y el MAPE, a medida que se realizan más pronósticos, tienden a ser mayores. Con base en la figura 6, los intervalos de pronóstico tienden a ser más amplios, resultado que es similar al que se conseguiría en un modelo de series de tiempo convencional. Además, se muestra que antes de una próxima caída del precio del dólar transcurren, en promedio, entre 3 y 6 días. 

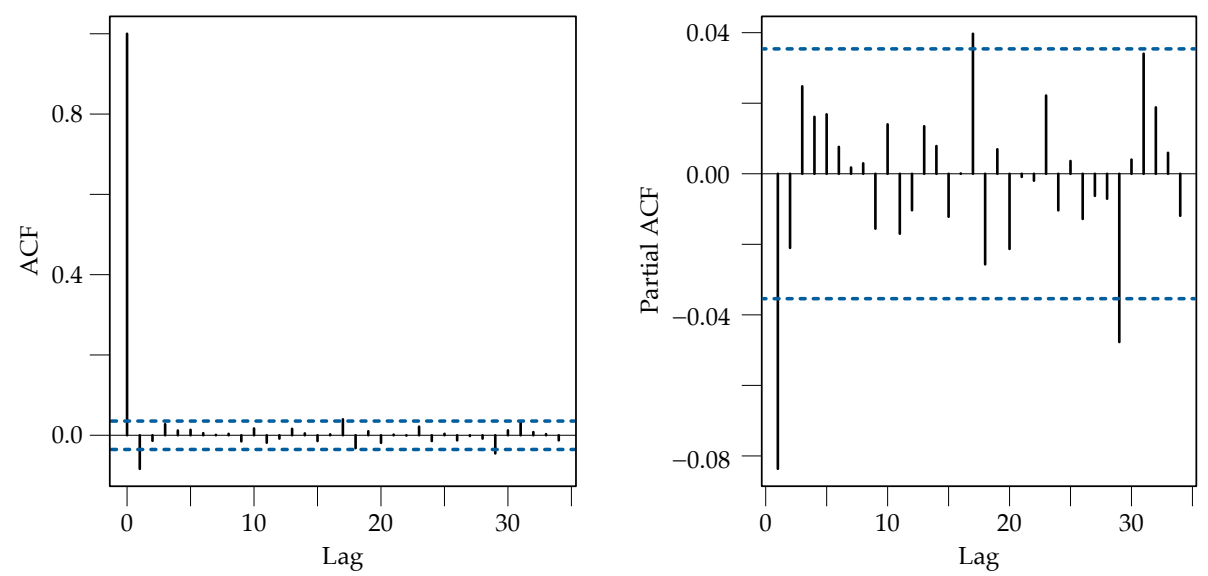

Figura 5. Función de autocorrelación y autocorrelación parcial de los residuales del modelo ACD $(1,1)$

\section{Conclusiones}

En este trabajo se propuso un modelo ADC con distribución de TRayleigh, aplicado al modelado y predicción de bajas en el dólar utilizando la TRM colombiana.

A partir de la ecuación estimada (5), se realizó el pronóstico de la duración esperada de una a cuatro posibles bajas del dólar en el mercado cambiario colombiano. Así, se encontró que el tiempo esperado hasta observar una próxima baja es de 3 a 6 días.

La estimación del parámetro $\lambda$ de la distribución de TRayleigh, asociada a los residuales del modelo, obtuvo un valor de 0.702 , que indica que el tiempo transcurrido hasta observar una baja en el dólar tiene exceso de curtosis y, en consecuencia, un comportamiento distribucional con asimetría marcada y cola larga. Este comportamiento distribucional de las duraciones es coherente con las propiedades de asimetría y curtosis mostradas por Dey, Raheem y Mukherjee (2017). El modelo propuesto muestra la estacionariedad existente en los días que transcurren hasta observar una baja en el dólar.

Con base en el trabajo de Pyrlik (2013) y por simulación del evento crítico, se fijaron, a partir de los valores de los log-retornos de la TRM por debajo de 0 , los eventos de caída del dólar. Los residuales del modelo cumplen con el supuesto de independencia. La RMSE y el MAPE muestran que conforme aumenta el número de pronósticos, el poder predictivo del modelo es menor. 


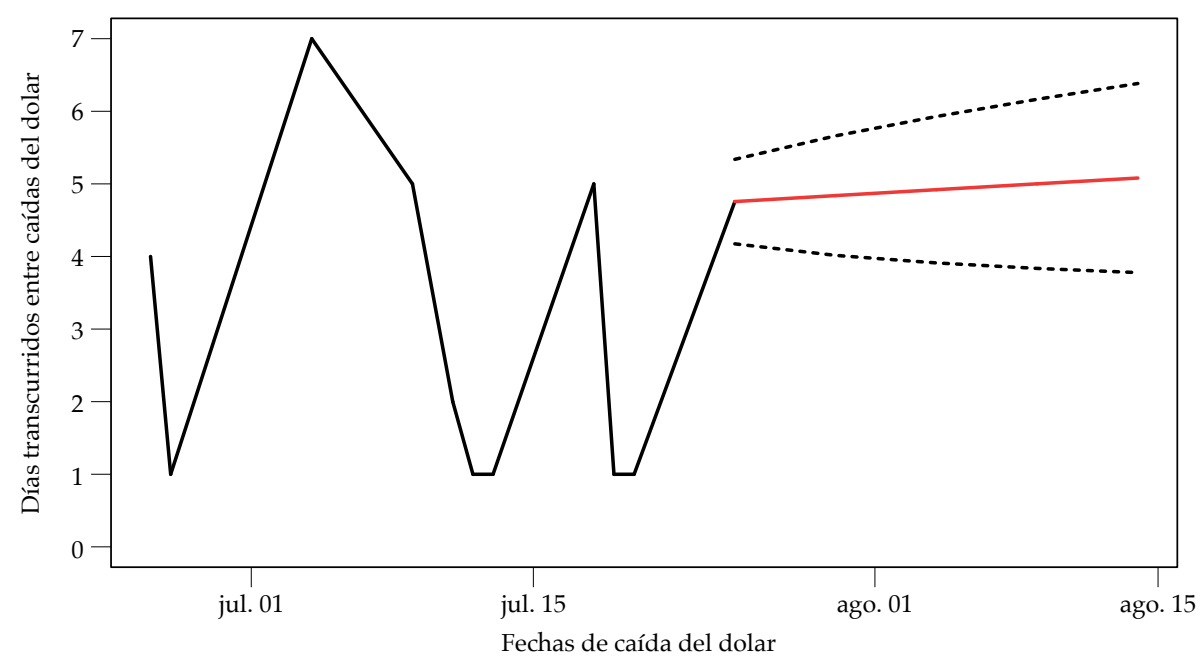

Figura 6. Pronósticos calculados del modelo ACD $(1,1)$

\section{Referencias}

Banco de la República. (1991-2020). Tasa representativa del mercado.Colombia. Recuperado de https://www.banrep.gov.co/es/estadisticas/trm

Bollerslev, T. (1986). Generalized autoregressive conditional heteroskedasticity. Journal of Economics, 31(3), 307-327.

Bonifaz, A. C. (2004). El dólar estadounidense: el impacto de sus fluctuaciones. Problemas del Desarrollo. Revista Latinoamericana de Economía, 35(136), 27-47.

Cheung, L. (2014). High frequency data: Modeling durations via the ACD and log ACD models. (Tesis de maestría, University of Connecticut).

Dey, S., Raheem, E., \& Mukherjee, S. (2017). Statistical properties and different methods of estimation of transmuted Rayleigh distribution. Revista Colombiana de Estadística, 40(1), 165-203.

Engle, R. F., \& Russell, J. R. (1997). Forecasting the frequency of changes in quoted foreign exchange prices with the autoregressive conditional duration model. Journal of empirical finance, 4(2-3), 187-212.

Engle, R. F., \& Russell, J. R. (1998). Autoregressive conditional duration: A new model for irregularly spaced transaction data. Econometrica, 66(5), 1127-1162.

Farmer, R. E. (2012). The stock market crash of 2008 caused the great recession: Theory and evidence. Journal of Economic Dynamics and Control, 36(5), 693-707. 
Henao, J. D. V., \& Rivera, L. M. G. (2006). Modelado del índice de tipo de cambio real colombiano usando redes neuronales artificiales. Cuadernos de Administración, 19(32), 319-336.

Jalil, M. A., \& Misas, M. (2007). Evaluación de pronósticos del tipo de cambio utilizando redes neuronales y funciones de pérdida asimétricas. Revista Colombiana de Estadística, 30(1), 143-161.

Tse, Y.-K., \& Yang, T. T. (2012). Estimation of high-frequency volatility: An autoregressive conditional duration approach. Journal of Business $\mathcal{E}$ Economic Statistics, 30(4), 533-545.

Liu, S., \& Tse, Y.-K. (2015). Intraday value-at-risk: An asymmetric autoregressive conditional duration approach. Journal of econometrics, 189(2), 437-446.

Merovci, F. (2013). Transmuted Rayleigh distribution. Austrian Journal of Statistics.

Alonso, J., \& Patiño, C. (2005). Determinantes de la tasa de cambio nominal en Colombia: evaluación de pronósticos. https://repository.icesi.edu.co/biblioteca_digital/bitstream/10906/81321/1/alonso_tasa_cambio_2005.pdf

Pyrlik, V. (2013). Autoregressive conditional duration as a model for financial market crashes prediction. Physica A: Statistical Mechanics and its Applications, 392(23), 6041-6051.

Santana, J. C. (2006). Predicción de series temporales con redes neuronales: una aplicación a la inflación colombiana. Revista Colombiana de Estadística, 29(1), 77-92.

Shapiro, S. S., Wilk, M. B., \& Chen, H. J. (1968), A comparative study of various tests for normality. Journal of the American Statistical Association, 63(324), 1343-1372.

Shaw, W. T., \& Buckley, I. R. (2009). The alchemy of probability distributions: Beyond gram-charlier expansions, and a skew-kurtotic-normal distribution from a rank transmutation map. Recuperado de arXiv.or (0901.0434v1).

Tsay, R. S. (2005), Analysis of Financial Time Series. Hoboken, N. J.: Wiley.

Tsay, R. S. (2011), Acd.cmle r function. Recuperado de https://faculty.chicagobooth.edu/ruey.tsay/teaching/introTS/acd.R 